\title{
Effects of Radiation and Cold Wall Temperature Boundary Conditions on Natural Convection in a Vertical Annular Porous Medium
}

\author{
M. Patil ${ }^{\dagger}$, P. G. Hegde and K. N. Seetharamu \\ Department of Mechanical Engineering, PES Institute of Technology, Bangalore- 560085, India \\ †Corresponding Author Email: patilmukesh@pes.edu
}

(Received February 26, 2011; accepted August 27, 2011)

\begin{abstract}
This paper deals with the numerical solution for natural convection in a vertical annular porous medium for various cold wall temperature boundary conditions and radiation parameters. The heat transfer is assumed to take place by convection and radiation. The inner wall (hot wall) of the annulus is maintained at an isothermal temperature while the outer wall (cold wall) is subjected to different temperature conditions. The temperature conditions maintained at the cold wall are evaluated for uniform as well as non uniform temperatures. The fluid is assumed to obey Darcy's law. The governing partial differential equations are non-dimensionalised and solved by finite element method. The porous medium is discretised with unstructured triangular elements. The effects of radius ratio and Rayleigh number on the Nusselt number and Sherwood number are investigated on the annulus for different temperatures at cold wall. The effects of radiation on flow patterns, temperature distribution and concentration distribution are discussed. The results reveal that the Nusselt number and Sherwood number at cold wall decrease with the increase in radius ratio, whereas they increase with the radius ratio at hot wall for different temperature boundary conditions at the cold wall. Temperature cold wall conditions have pronounced effect on the Nusselt and Sherwood numbers.
\end{abstract}

Keywords: Radiation, convection, numerical method, porous medium, vertical annulus, heat transfer.

\section{NOMENCLATURE}

$\begin{array}{ll}\mathrm{A} & \text { area, } \mathrm{m}^{2} \\ \mathrm{~A}_{\mathrm{r}} & \text { aspect ratio }=\frac{\mathrm{H}}{\mathrm{L}_{\mathrm{ref}}} \\ \mathrm{Avg} . & \text { average } \\ \mathrm{C} & \text { concentration factor } \\ \overline{\mathrm{C}} & \text { non dimensional concentration factor } \\ \mathrm{C}_{\mathrm{p}} & \text { specific heat, } \mathrm{Jg}^{-1} \mathrm{~K}^{-1} \\ \mathrm{D}_{\mathrm{p}} & \text { particle diameter, } \mathrm{m} \\ \mathrm{g} & \text { gravitational acceleration, } \mathrm{ms}^{-1} \\ \mathrm{~h} & \text { heat transfer co-efficient, } \mathrm{Wm}^{-2} \mathrm{~K}^{-1} \\ \mathrm{H} & \text { height of the annulus, } \mathrm{m} \\ \mathrm{i} & \text { inner wall } \\ \mathrm{K} & \text { permeability of porous medium } \\ \mathrm{Le} & \text { Lewis number } \\ \mathrm{L}_{\mathrm{ref}} & =\left(\mathrm{r}_{\mathrm{o}}-\mathrm{r}_{\mathrm{i}}\right), \mathrm{m} \\ \mathrm{N} & \text { shape function } \\ \mathrm{Nu} . & \text { Nusselt number } \\ \overline{\mathrm{Nu}} & \text { average Nusselt number } \\ \mathrm{o} & \text { outer wall } \\ \mathrm{r}, \mathrm{z} & \text { cylindrical co-ordinates } \\ \overline{\mathrm{r}}, \overline{\mathrm{z}} & \text { non dimensional cylindrical co-ordinates } \\ \mathrm{ref} & \text { reference }\end{array}$

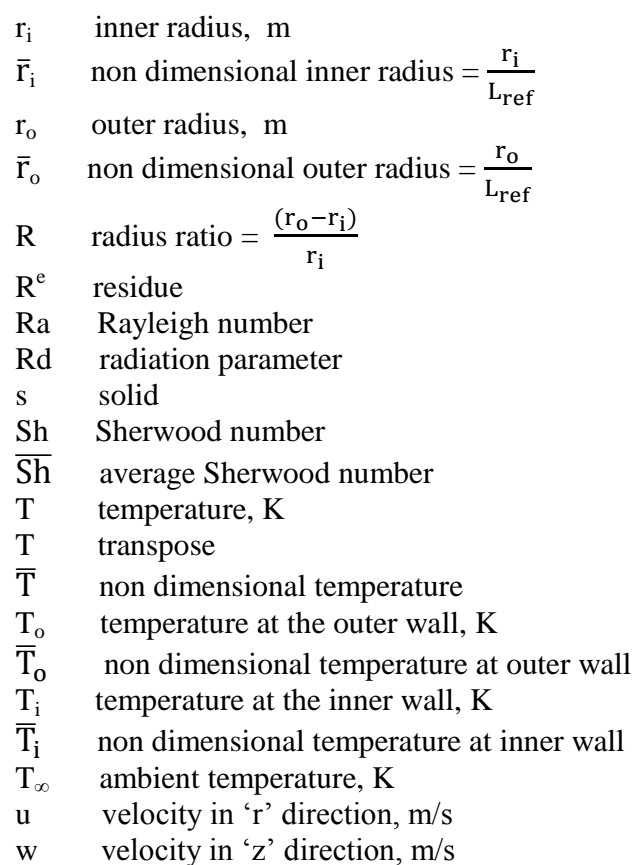


w wall

$\alpha \quad$ thermal diffusivity, $\mathrm{m}^{2} \mathrm{~s}^{-1}$

$\beta_{c} \quad$ co-efficient of concentration expansion, $\mathrm{K}^{-1}$

$\beta_{\mathrm{R}}$ rosseland extinction co-efficient

$\beta_{\mathrm{T}} \quad$ co-efficient of thermal expansion, $\mathrm{K}^{-1}$

$\Delta$ potential difference

$v$ kinematic viscosity, $\mathrm{m}^{2} \mathrm{~s}^{-1}$

$\kappa$ thermal conductivity, $\mathrm{Wm}^{-1} \mathrm{~K}^{-1}$

$\rho$ density, $\mathrm{kg} \mathrm{m}^{-3}$

$\lambda$ power law exponent

\section{INTRODUCTION}

In recent years, natural convection, heat and mass transfer through porous medium have received remarkable interest among the researchers. Porous medium plays important role in many geophysical and engineering applications such as energy recovery from petroleum resources, thermal insulation of buildings, chemical reactors, nuclear waste disposal etc. When the convection is comparatively small, radiation plays a vital role and hence it cannot be neglected. A comprehensive literature review on convection in a fluid-saturated porous medium may be found in the books by Nield and Bejan (1999) and Ingham and Pop (2005). Reda (1986) has carried out the experimental investigation of a finite vertical cylinder. Here the inner cylinder had a finite length heat source whereas the outer cylinder was maintained at constant temperature. The results showed that the radial temperature drop across the annulus was found to systematically depart from the finite-length cylinder as heat power was increased. Muralidhar (1989) has analyzed the mixed convection in a vertical annulus. The study here was carried out for height to gap ratio of 10 and outer to inner radius ratio of 2 . It was noticed that the forced convection dominates in the first $10 \%$ of entry length region. Rajamani et al. (1995) have studied the natural convective heat transfer in an annular cylinder embedded with porous medium by making use of finite element method and discussed the effect of aspect ratio and radius ratio of the annulus on the heat transfer rate. Hossain and Alim (1997) have investigated the natural convection radiation interaction on boundary layer flow along vertical cylinder by using local non-similarity method and implicit finite difference scheme with Keller box elimination method. Raptis (1998) has investigated the heat transfer behavior of vertical plate in porous medium subjected to constant suction velocity. Inspired by electric heating elements, Wang (1998) has given a set of critical Rayleigh numbers for various size ratios of cylinder at which the convection heat transfer starts. Here the bottom surfaces of the cylinder were subjected to constant heat flux with the side walls being insulated. The effect of radiation on natural convection over a vertical cylinder embedded with porous medium has been studied by Yih (1999). He has employed the finite difference method and has presented the dimensionless temperature profile and $\mathrm{N}_{\mathrm{u}} / \mathrm{R}_{\mathrm{a}}{ }^{1 / 2}$ for different parameters. Cherif and Sifaoui (2004) have considered radiation along with conduction and convection to predict the heat transfer behavior in a cylindrical enclosure. The problem of heat transfer in saturated porous vertical annulus with combined effect of radiation and convection has been studied for the case of $\overline{\mathrm{T}}_{\mathrm{o}}=0$ only by Irfan et al. (2006). Heat transfer
$\Phi$ Porosity

$\mu \quad$ dynamic viscosity, $\mathrm{m}^{2} \mathrm{~s}^{-1}$

$\sigma \quad$ Stephan Boltzmann constant

$\Psi \quad$ stream function $\mathrm{W} / \mathrm{m}^{2} \mathrm{~K}^{4}$

$\bar{\Psi}$ non dimensional stream function

$\infty$ conditions at outer radius

analysis of porous medium in a conical cylinder with variable wall temperature has been carried out by Ahmed et al. (2008). They investigated the fluid flow behavior in a complex geometry such as conical cylinder having variable wall temperature and concluded that the effect of cone angle on heat transfer behavior is significant. Srivastava and Singh (2010) have analyzed the mixed convection in a composite system bounded by vertical walls. They concluded that the velocity intensifies with the temperature potential between walls and reduces with the increase in viscosity ratio. The problem of heat transfer in porous annular cylinder requires attention as it has many practical applications such as gas cooled reactor vessels, bio-mass converters, insulated pipe lines etc.

Yet, to the best knowledge of the authors there appears to be no study in the literature concerned with the combined effect of natural convection and radiation on a saturated vertical annular porous medium with different temperature cold wall conditions $\left(\overline{\mathrm{T}}_{\mathrm{o}}\right)$. The work carried out in the past corresponds to the case of $\overline{\mathrm{T}}_{0}=0$ as observed from the literature. In the present work, three case studies have been carried out. The first two cases correspond to two uniform non dimensional temperatures $\left(\overline{\mathrm{T}}_{\mathrm{o}}=0.1\right.$ and 0.9$)$ at the cold wall boundary condition for the annulus. In the third case the effect of non uniform temperature variation in accordance with the power law, along the cold wall of the vertical annulus is analyzed.

The objective of the present work is to study the effect of radiation on natural convection, heat and mass transfer, when the temperatures boundary conditions at the cold wall of the porous annulus are varied. The effects of radius ratio and Rayleigh number on the heat and mass transfer in terms of average Nusselt number and average Sherwood number respectively are analyzed. Also their effects on temperature, flow and concentration distributions are examined.

\section{MATHEMATICAL FORMULATION}

The In the present study, a saturated vertical porous annulus of inner radius $\left(r_{i}\right)$ and outer radius $\left(r_{o}\right)$ is considered. Let the inner wall (hot wall) be at temperature $\mathrm{T}_{\mathrm{i}}$ and outer wall (cold wall) be at temperature $T_{o}$ such that $T_{o}<T_{i}$. The two horizontal walls are insulated, thus making them adiabatic. Figure 1 shows the cross section of the vertical porous annulus considered in the present work. 


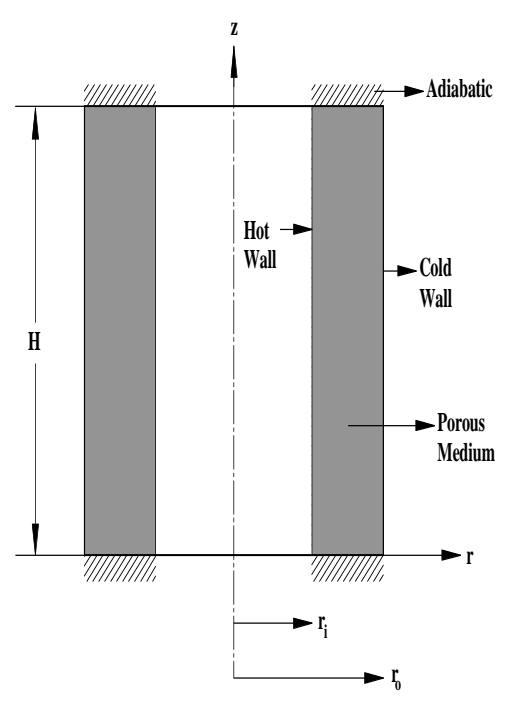

Fig. 1. Cross section of the axi- symmetric vertical annular porous medium

The $\mathrm{r}$ and $\mathrm{z}$ co ordinates point towards the radius and height of the vertical porous annulus respectively.

The following assumptions are made in the present study:

a) Porous medium is saturated with fluid

b) The fluid is assumed to be gray emitting but non-scattering

c) The fluid and medium are in local thermal equilibrium in the domain

d) The porous medium is isotropic and homogeneous

e) Fluid properties are constant except for the variation of density

f) Darcy's law is assumed for the flow in the porous medium

With these assumptions, the governing equations can be written as:

Continuity equation:

$\frac{\partial(\mathrm{ru})}{\partial \mathrm{r}}+\frac{\partial(\mathrm{rw})}{\partial \mathrm{z}}=0$

Momentum equation:

$\frac{\partial \mathrm{w}}{\partial \mathrm{r}}-\frac{\partial \mathrm{u}}{\partial \mathrm{z}}=\frac{\mathrm{gK} \beta}{\mathrm{v}} \frac{\partial \mathrm{T}}{\partial \mathrm{r}}$

where

$$
\mathrm{K}=\frac{\left(\mathrm{D}_{\mathrm{p}}^{2} \Phi^{3}\right)}{180\left((1-\Phi)^{2}\right)}
$$

The variation of density with respect to temperature can be described by Boussinesq approximation as:

$\rho=\rho_{\infty}\left[1-\beta\left(\mathrm{T}-\mathrm{T}_{\infty}\right)\right]$

Energy equation is written as:

$$
\begin{aligned}
& \rho \mathrm{C}_{\mathrm{p}}\left(\mathrm{u} \frac{\partial \mathrm{T}}{\partial \mathrm{r}}+\mathrm{w} \frac{\partial \mathrm{T}}{\partial \mathrm{z}}\right)= \\
& {\left[\alpha\left\{\frac{1}{\mathrm{r}} \frac{\partial}{\partial \mathrm{r}}\left(\mathrm{r} \frac{\partial \mathrm{T}}{\partial \mathrm{r}}\right)+\frac{\partial^{2} \mathrm{~T}}{\partial \mathrm{z}^{2}}\right\}-\frac{1}{\mathrm{r}} \frac{\partial}{\partial \mathrm{r}}\left(\mathrm{rq}_{\mathrm{r}}\right)\right]}
\end{aligned}
$$

Invoking Rosseland approximation for radiation: (Hossain, 1997 and Yih, 1999).

where

$\mathrm{q}_{\mathrm{r}}=\frac{4 \mathrm{n}^{2} \sigma}{3 \beta_{\mathrm{R}}} \frac{\partial \mathrm{T}^{4}}{\partial \mathrm{r}}$

The continuity equation can be solved by introducing the stream function $(\Psi)$ as:

$$
\begin{aligned}
& \mathrm{u}=-\frac{1}{\mathrm{r}} \frac{\partial \Psi}{\partial \mathrm{z}} \\
& \mathrm{w}=\frac{1}{\mathrm{r}} \frac{\partial \Psi}{\partial \mathrm{r}}
\end{aligned}
$$

Concentration equation is written as:

$u \frac{\partial \mathrm{C}}{\partial \mathrm{r}}+\mathrm{w} \frac{\partial \mathrm{C}}{\partial \mathrm{z}}=\mathrm{D}\left\{\frac{1}{\mathrm{r}} \frac{\partial}{\partial \mathrm{r}}\left(\mathrm{r} \frac{\partial \mathrm{C}}{\partial \mathrm{r}}\right)+\frac{\partial^{2} \mathrm{C}}{\partial \mathrm{z}^{2}}\right\}$

The boundary conditions used are:

at $\mathrm{r}=\mathrm{r}_{\mathrm{i}}, \mathrm{T}=\mathrm{T}_{\mathrm{w}}, \mathrm{C}=\mathrm{C}_{\mathrm{i}},=0$

at $\mathrm{r}=\mathrm{r}_{\mathrm{o}}, \mathrm{T}=\mathrm{T}_{\mathrm{o}}, \mathrm{C}=0,=0$

The following non-dimensional parameters have been used:

$\bar{r}=\frac{r}{L_{\text {ref }}}, \bar{z}=\frac{\mathrm{z}}{\mathrm{L}_{\mathrm{ref}}}, \bar{\Psi}=\frac{\Psi}{\alpha \mathrm{L}_{\mathrm{ref}}}$,

$\bar{T}=\frac{\left(\mathrm{T}_{\mathrm{o}}-\mathrm{T}_{\infty}\right)}{\left(\mathrm{T}_{\mathrm{w}}-\mathrm{T}_{\infty}\right)}, R d=\frac{4 \sigma \mathrm{T}_{\infty}^{3}}{\beta_{\mathrm{R}} \kappa}$,

$R a=\frac{\left(\mathrm{g} \beta_{\mathrm{T}} \Delta \mathrm{TKLref}\right)}{v \alpha}, L e=\frac{\alpha}{\mathrm{D}}, N=\frac{\left(\beta_{\mathrm{c}} \Delta \mathrm{C}\right)}{\left(\beta_{\mathrm{T}} \Delta \mathrm{T}\right)}$,

$\bar{C}=\frac{\left(\mathrm{C}-\mathrm{C}_{\infty}\right)}{\left(\mathrm{C}_{\mathrm{w}}-\mathrm{C}_{\infty}\right)}$

Thermal diffusivity $(\alpha)$ belongs to effective porous medium and is defined as:

$\alpha=\frac{\mathrm{k}}{\rho \mathrm{C}_{\mathrm{p}}}$

The boundary conditions used in terms of the nondimensional parameters are:

at $r=r_{i}, \bar{T}=\bar{T}_{i}=1, \overline{\mathrm{C}}=1, \bar{\Psi}=0$

at $r=r_{o}, \bar{T}=\bar{T}_{o}=\frac{\left(T_{o}-T_{\infty}\right)}{\left(T_{w}-T_{\infty}\right)}, \overline{\mathrm{C}}=0, \bar{\Psi}=0$

The non-dimensional parameters from Eqs. (13a) and (13b) are substituted in Eqs. (2), (5) and (9). The nondimensionalised forms of the equations Eqs. (2), (5) and (9) are as follows:

Non-dimensionalised momentum equation: 


$$
\frac{\partial^{2} \bar{\Psi}}{\partial \bar{y}^{2}}+\bar{r} \frac{\partial}{\partial \bar{r}}\left(\frac{1}{\bar{r}} \frac{\partial \bar{\Psi}}{\partial \bar{r}}\right)=\bar{r} R a\left[\frac{\partial \bar{T}}{\partial \bar{r}}+N \frac{\partial \bar{C}}{\partial \bar{r}}\right]
$$

Non-dimensionalised energy equation:

$\frac{1}{\bar{r}}\left[\frac{\partial \bar{\Psi}}{\partial \bar{r}} \frac{\partial \bar{T}}{\partial \bar{z}}-\frac{\partial \bar{\Psi}}{\partial \bar{z}} \frac{\partial \bar{T}}{\partial \bar{r}}\right]=\frac{1}{\bar{r}} \frac{\partial}{\partial \bar{r}}\left(\left(1+\frac{4 \mathrm{Rd}}{3}\right) \bar{r} \frac{\partial \bar{T}}{\partial \bar{r}}\right)+\frac{\partial^{2} \bar{T}}{\partial \bar{z}^{2}}$

Non-dimensionalised concentration equation:

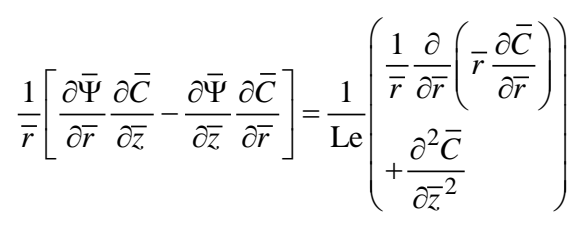

When $\mathrm{Rd}=0$, the case reduces to that of pure natural convection for the given vertical annular porous medium (Rajamani et al. 1995).

In the present study, $\overline{\mathrm{T}}_{\mathrm{o}}$ (Eq. (13b)) has been evaluated for four values. They are $0.1,0.4,0.8$ and 0.9 . They represent the uniform non-dimensional temperature at the cold wall. However taking the length of this paper into consideration, only two cases of $\bar{T}_{0}=0.1$ and 0.9 have been presented. The two cases aid in determination of the natural convection, heat and mass transfer for uniform temperature boundary conditions in case of the vertical annular porous medium considered. It is observed from Fig. 2 that $\overline{\mathrm{Nu}}$ increases almost linearly with the radiation parameter for all the boundary conditions of $\overline{\mathrm{T}}_{\mathrm{o}}$ on the cold wall. The value of $\overline{\mathrm{Nu}}$ is around unity at $\overline{\mathrm{T}}_{\mathrm{O}}=0.4$ and further decreases as the value of $\bar{T}_{0}$ increases. At $\bar{T}_{0}=0.9$, the value of $\overline{\mathrm{Nu}}$ is near to zero, indicating that the heat transfer reduces and ceases to exist as $\overline{\mathrm{T}}_{\mathrm{o}}$ approaches unity.

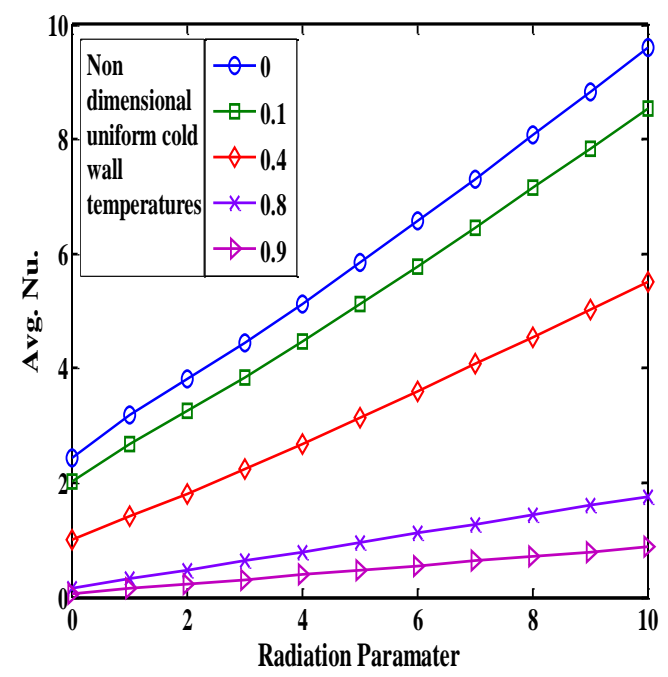

Fig. 2. Variation of $\overline{\mathbf{N u}}$ with $\mathrm{Rd}$

Further, the cold wall temperature is assumed to be non uniform, obeying the power law function. It varies in the vertical direction such that $\mathrm{T}_{\mathrm{o}}>\mathrm{T}_{\infty}$. For the problem under investigation the boundary conditions can be defined as:

$$
\begin{aligned}
& \text { at } \quad r=r_{i}, \bar{T}=\bar{T}_{i}=1, \bar{C}=1, \bar{\Psi}=0 \\
& \text { at } r=r_{o}, \bar{T}=\bar{T}_{o}=\left[\mathrm{B}(\mathrm{z})^{\lambda}\right], \bar{C}=0, \bar{\Psi}=0
\end{aligned}
$$

In Eq. (17b), B is a constant. In the present study, $\lambda$ has been evaluated for a value of 0.25

\section{NUMERICAL METHOD}

Equations (14) - (16) are the coupled partial differential equations, to be solved in order to predict the fluid flow, heat and mass transfer behavior. These equations are solved by using finite element method. A simple three noded triangular element is considered. $\overline{\mathrm{T}}, \bar{\psi}$ and $\overline{\mathrm{C}}$ varying inside the element can be expressed as:

$$
\begin{aligned}
& \bar{T}=N_{1} \overline{\mathrm{T}}_{1}+N_{2} \overline{\mathrm{T}}_{2}+N_{3} \overline{\mathrm{T}}_{3} \\
& \bar{\Psi}=N_{1} \bar{\Psi}_{1}+N_{2} \bar{\Psi}_{2}+N_{3} \bar{\Psi}_{3} \\
& \bar{C}=N_{1} \bar{C}_{1}+N_{2} \bar{C}_{2}+N_{3} \bar{C}_{3}
\end{aligned}
$$

$\mathrm{N}_{1}, \mathrm{~N}_{2}$ and $\mathrm{N}_{3}$ are the shape functions expressed in general as:

$$
N_{m}=\frac{\mathrm{a}_{\mathrm{m}}+\mathrm{b}_{\mathrm{m}} \mathrm{x}+\mathrm{c}_{\mathrm{m}} \mathrm{y}}{2 \mathrm{~A}} \text {, where } \mathrm{m}=1,2,3 .
$$

Details of finite element formulation can be obtained from the work done by Segerlind (1982) and Lewis et al. (2004).

The coupled matrix equations are obtained after applying Galerkin method to Eqs. (14) - (16). The matrix equations are assembled to get the global matrix for the whole domain, which is solved iteratively to obtain $\overline{\mathrm{T}}, \overline{\mathrm{C}}$ and $\bar{\psi}$ in the porous medium. In order to get accurate results, the tolerance levels of the solutions for $\overline{\mathrm{T}}, \overline{\mathrm{C}}$ and $\bar{\psi}$ are set at $10^{-5}, 10^{-7}$ and $10^{-9}$ respectively. Element size in the domain is varied by having large number of elements located near the wall, where large variations in $\bar{T}, \overline{\mathrm{C}}$ and $\bar{\psi}$ are expected. A sufficiently dense mesh is chosen to make the solution invariant. In the present study a mesh size consisting of 1800 elements and 961 nodes is used to solve the governing equations.

Applying Galerkin method to Eq. (14) one can obtain:

$$
\begin{aligned}
\left\{\mathrm{R}^{\mathrm{e}}\right\}=-\int_{\mathrm{A}} \mathrm{N}^{\mathrm{T}}[ & \frac{\partial^{2} \bar{\Psi}}{\partial \overline{\mathrm{C}}^{2}}+\overline{\mathrm{r}} \frac{\partial}{\partial \overline{\mathrm{r}}}\left(\frac{1}{\overline{\mathrm{r}}} \frac{\partial \bar{\Psi}}{\partial \overline{\mathrm{r}}}\right) \\
& -\overline{\mathrm{r} R a}\left(\frac{\partial \overline{\mathrm{T}}}{\partial \overline{\mathrm{r}}}\right. \\
& \left.\left.+\mathrm{N} \frac{\partial \overline{\mathrm{C}}}{\partial \overline{\mathrm{r}}}\right)\right] 2 \pi \overline{\mathrm{r}} \mathrm{dA}=0
\end{aligned}
$$

Applying Galerkin method to Eq. (15) one can obtain: 
$\left\{\mathrm{R}^{\mathrm{e}}\right\}$

$$
\begin{aligned}
& =\int_{\mathrm{A}} \mathrm{N}^{\mathrm{T}}\left[\frac{1}{\overline{\mathrm{r}}}\left[\frac{\partial \bar{\Psi}}{\partial \overline{\mathrm{r}}} \frac{\partial \overline{\mathrm{T}}}{\partial \overline{\mathrm{Z}}}-\frac{\partial \bar{\Psi}}{\partial \overline{\mathrm{Z}}} \frac{\partial \overline{\mathrm{T}}}{\partial \overline{\mathrm{r}}}\right]\right. \\
& \left.-\left(\frac{1}{\overline{\mathrm{r}}} \frac{\partial}{\partial \overline{\mathrm{r}}}\left(\left(1+\frac{4 \mathrm{Rd}}{3}\right) \overline{\mathrm{r}} \frac{\partial \overline{\mathrm{T}}}{\partial \overline{\mathrm{r}}}\right)+\frac{\partial^{2} \overline{\mathrm{T}}}{\partial \overline{\mathrm{Z}}^{2}}\right)\right] \\
& 2 \pi \overline{\mathrm{r}} \mathrm{d} \mathrm{A}=0
\end{aligned}
$$

Applying Galerkin method to Eq. (16) one can obtain:

$$
\begin{aligned}
\left\{\mathrm{R}^{\mathrm{e}}\right\}=-\int_{\mathrm{A}} \mathrm{N}^{\mathrm{T}}[ & \frac{1}{\overline{\mathrm{r}}}\left[\frac{\partial \bar{\Psi}}{\partial \overline{\mathrm{r}}} \frac{\partial \overline{\mathrm{C}}}{\partial \overline{\mathrm{Z}}}-\frac{\partial \bar{\Psi}}{\partial \overline{\mathrm{Z}}} \frac{\partial \overline{\mathrm{C}}}{\partial \overline{\mathrm{r}}}\right] \\
& -\frac{1}{\mathrm{Le}}\left(\frac{1}{\overline{\mathrm{r}}} \frac{\partial}{\partial \overline{\mathrm{r}}}((1\right. \\
& \left.\left.+\frac{4 \mathrm{Rd}}{3}\right) \overline{\mathrm{r}} \frac{\partial \overline{\mathrm{C}}}{\partial \overline{\mathrm{r}}}\right) \\
& \left.\left.+\frac{\partial^{2} \overline{\mathrm{C}}}{\partial \overline{\mathrm{z}}^{2}}\right)\right] 2 \pi \overline{\mathrm{r}} \mathrm{A} \mathrm{A}=0
\end{aligned}
$$

\section{RESUlt AND Discussion}

In the first two cases the cold wall is subjected to different, uniform temperature boundary conditions; $\overline{\mathrm{T}}_{\mathrm{O}}=0.1$ and 0.9 respectively. In the third case, analysis is made on the variation of non uniform temperature effect along the cold wall of the vertical annulus. The variation of the non uniform temperature is in accordance with the power law. The hot wall of the annulus is maintained at a constant isothermal temperature in all the three cases $\left(\bar{T}_{i}=1\right)$. In the present study the value of the power law exponent $(\lambda)$ considered is 0.25 (Eq. (17b)). Results are obtained in terms of average Nusselt number $(\overline{\mathrm{Nu}})$ and average Sherwood number $(\overline{\mathrm{Sh}})$.

Average Nusselt number and Sherwood number are given by:

$\overline{\mathrm{Nu}}_{\text {hot }}=-\frac{\int_{0}^{\bar{z}}\left[\left(1+\frac{4 \mathrm{Rd}}{3}\right) \frac{\partial \overline{\mathrm{T}}}{\partial \overline{\mathrm{r}}}\right]_{\overline{\mathrm{r}}=\overline{\mathrm{r}}_{\mathrm{i}}} \mathrm{d} \overline{\mathrm{z}}}{\overline{\mathrm{z}}}$

$\overline{\mathrm{Nu}}_{\text {cold }}=-\frac{\int_{0}^{\overline{\mathrm{z}}}\left[\left(1+\frac{4 \mathrm{Rd}}{3}\right) \frac{\partial \overline{\mathrm{T}}}{\partial \overline{\mathrm{r}}}\right]_{\overline{\mathrm{r}}=\overline{\mathrm{r}}_{\mathrm{o}}} \mathrm{d} \overline{\mathrm{z}}}{\overline{\mathrm{z}}}$

$\overline{\operatorname{Sh}}_{\text {hot }}=-\frac{\int_{0}^{\bar{z}}\left[\frac{\partial \overline{\bar{c}}}{\partial \overline{\mathrm{r}}}\right]_{\overline{\mathrm{r}}=\overline{\mathrm{r}}_{\mathrm{i}}} \mathrm{d} \overline{\mathrm{z}}}{\overline{\mathrm{z}}}$

$\overline{\mathrm{Sh}}_{\text {cold }}=-\frac{\int_{0}^{\overline{\mathrm{z}}}\left[\frac{\partial \overline{\mathrm{C}}}{\partial \overline{\mathrm{r}}}\right]_{\overline{\mathrm{r}}=\overline{\mathrm{r}}_{\mathrm{O}}} \mathrm{d \overline { \textrm {z } }}}{\overline{\mathrm{z}}}$

The temperature gradients $\left(\frac{\partial \overline{\mathrm{T}}}{\partial \overline{\mathrm{r}}}\right)$ in Eqs. (25) - (28) are evaluated using a 4-point polynomial along the nodes near the hot and cold walls of the vertical annulus.

\subsection{Verification Cases}

In order to verify the accuracy of the present method of numerical analysis using Finite Element Method, $\overline{\mathrm{Nu}}$ for different values of $\mathrm{R}$ is compared with those available in the literature (Irfan et al., 2006) The comparison is shown in Figs. 3(a) and 3(b), at $\mathrm{Ra}=100$ for three different values of $\operatorname{Rd}(0,0.5$ and 1$)$. It is evident from these figures that the present method has good accuracy in predicting the heat transfer behavior of the vertical porous annulus as compared with those available in the literature.

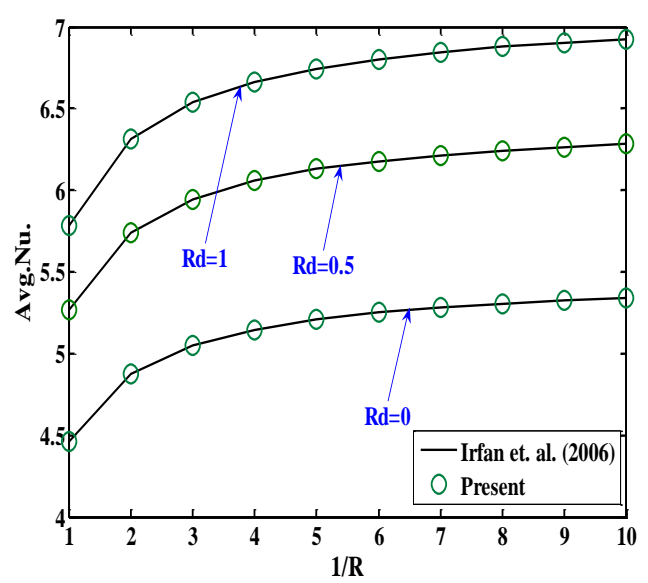

(a)

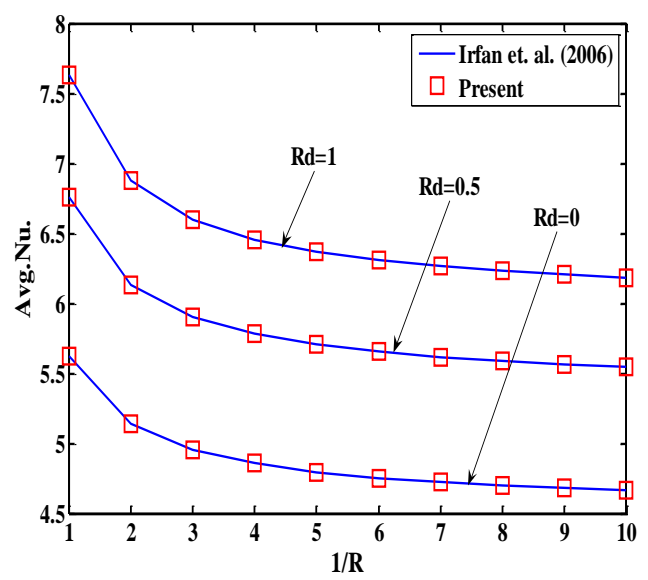

(b)

Fig. 3. (a). Variation of $\overline{\mathbf{N u}}$ with $1 / \mathrm{R}$ for hot wall condition (Case A). (b). Variation of $\overline{\mathbf{N u}}$ with $1 / \mathrm{R}$ for cold wall condition (Case B)

\section{Case A}

Figure 3(a) shows the variation of $\overline{\mathrm{Nu}}$ with $1 / \mathrm{R}$. It is observed that $\overline{\mathrm{Nu}}$ is considerably augmented when radiation comes into picture. The heat transfer is considerably high due to the combined effect of convection and radiation as compared to the case when the radiation does not exist i.e. pure natural convection $(\mathrm{Rd}=0)$. It is further observed that the decrease in radius ratio leads to decrease in $\overline{\mathrm{Nu}}$ at the hot wall due to the reduction in temperature gradient.

\section{Case B}

Figure 3(b) shows the variation of $\overline{\mathrm{Nu}}$ at cold wall of the annulus at given conditions. At the cold wall $\overline{\mathrm{Nu}}$ increases initially with the decrease in $\mathrm{R}$, but attains a constant value at lower values of $R$. 


\section{Parametric Cases}

In the present study, two parametric cases have been investigated. The effects of several parameters like radius ratio, radiation parameter, Rayleigh number and cold wall temperatures (uniform and non uniform) on the Nusselt and Sherwood numbers have been investigated for both hot and cold walls. The parameters considered in common to both the cases are as follows:

a) $\mathrm{R}=1$ to 10

b) $\mathrm{N}=1$

c) $\mathrm{Le}=1$

d) $\mathrm{A}_{\mathrm{r}}=5$

e) Cold wall temperatures:

i. Uniform temperatures: $\overline{\mathrm{T}}_{\mathrm{o}}=0.1,0.9$

ii. Non uniform temperatures: $\lambda=0.25$ (Eq. (17b))

\section{Case 1- Effect of Ra on $\overline{\mathrm{Nu}}$ and $\overline{\mathrm{Sh}}$}

This case shows variation of $\overline{\mathrm{Nu}}$ and $\overline{\mathrm{Sh}}$ against $\mathrm{R}$ for various values of $\mathrm{Ra}$ at the hot wall and cold wall, corresponding to the cold wall boundary conditions. Three different values considered for the Rayleigh number for the present case are: 10, 25 and 50 .

Figure 4(a) shows variation of $\overline{\mathrm{Nu}}$ and $\overline{\mathrm{Sh}}$ against $\mathrm{R}$ for various values of $\mathrm{Ra}$ at the hot wall, corresponding to the cold wall boundary condition with $\overline{\mathrm{T}}_{0}=0.1$. It is observed that $\overline{\mathrm{Nu}}$ and $\overline{\mathrm{Sh}}$ increase with $\mathrm{R}$. The variation of $\overline{\mathrm{Nu}}$ and $\overline{\mathrm{Sh}}$ is found to be almost linear. As seen from the Fig. 4(a), the effect of $\mathrm{Ra}$ on $\overline{\mathrm{Nu}}$ is significant due to the higher temperature potential between hot wall and the cold wall $(\Delta \overline{\mathrm{T}}=0.9)$. The effect of Ra on $\overline{\mathrm{Sh}}$ depends on the velocity only and it is found to have a significant effect on $\overline{\mathrm{Sh}}$. The temperature potential represented in the present work is calculated as follows:

$\Delta \overline{\mathrm{T}}=\left(\overline{\mathrm{T}}_{\mathrm{i}}-\overline{\mathrm{T}}_{\mathrm{o}}\right)$;

Thus for Case 1: $\Delta \overline{\mathrm{T}}=(1-0.1)=0.9$

Figure 4(b) shows variation of $\overline{\mathrm{Nu}}$ and $\overline{\mathrm{Sh}}$ against $\mathrm{R}$ for various values of $\mathrm{Ra}$ at the hot wall, corresponding to the cold wall boundary condition with $\overline{\mathrm{T}}_{\mathrm{o}}=0.9$. It is observed that $\overline{\mathrm{Nu}}$ and $\overline{\mathrm{Sh}}$ increase with R. However the increase in the value of $\overline{\mathrm{Nu}}$ is less due to the lower temperature potential between hot wall and the cold wall $(\Delta \overline{\mathrm{T}}=0.1)$. The variation of $\overline{\mathrm{Nu}}$ and $\overline{\mathrm{Sh}}$ is found to be almost linear. The effect of $\mathrm{Ra}$ on $\overline{\mathrm{Nu}}$ is not significant. However Ra has a significant effect on $\overline{\mathrm{Sh}}$ similar to the case of $\overline{\mathrm{T}}_{0}=0.1$.

Figure 4(c) shows variation of $\overline{\mathrm{Nu}}$ and $\overline{\mathrm{Sh}}$ against $\mathrm{R}$ for various values of $\mathrm{Ra}$ at the hot wall, corresponding to the non uniform temperature cold wall boundary condition with $\lambda=0.25$ (Eq. (17b)). $\overline{\mathrm{Nu}}$ and $\overline{\mathrm{Sh}}$ increase with $\mathrm{R}$. The variation of $\overline{\mathrm{Nu}}$ and $\overline{\mathrm{Sh}}$ with $\mathrm{R}$ is found to be almost linear. However their values in this case lie in between the values at hot wall corresponding to the cold wall boundary condition of $\overline{\mathrm{T}}_{\mathrm{o}}=0.1$ and 0.9 . The effect of $\mathrm{Ra}$ on $\overline{\mathrm{Nu}}$ is not significant in this case, however it has the similar influence on $\overline{\mathrm{Sh}}$ as in the previous cases corresponding to $\overline{\mathrm{T}}_{\mathrm{o}}=0.1$ and 0.9 .
Figure 4(d) shows variation of $\overline{\mathrm{Nu}}$ and $\overline{\mathrm{Sh}}$ against $\mathrm{R}$ for various values of $\mathrm{Ra}$ at the cold wall, corresponding to the cold wall boundary condition with $\overline{\mathrm{T}}_{\mathrm{o}}=0.1 . \overline{\mathrm{Nu}}$ and $\overline{\mathrm{Sh}}$ decrease with the increase in $\mathrm{R}$. The variation of $\overline{\mathrm{Nu}}$ and $\overline{\mathrm{Sh}}$ is found to be non linear. As seen from the Fig. 4(d), the effect of $\mathrm{Ra}$ on $\overline{\mathrm{Nu}}$ is significant due to the higher temperature potential between hot wall and the cold wall $(\Delta \overline{\mathrm{T}}=0.9)$.

Figure 4(e) shows variation of $\overline{\mathrm{Nu}}$ and $\overline{\mathrm{Sh}}$ against $\mathrm{R}$ for various values of $\mathrm{Ra}$ at the cold wall, corresponding to the cold wall boundary condition with $\overline{\mathrm{T}}_{\mathrm{o}}=0.9$. It is observed that $\overline{\mathrm{Nu}}$ and $\overline{\mathrm{Sh}}$ decrease with the increase in $\mathrm{R}$. The variation of $\overline{\mathrm{Nu}}$ and $\overline{\mathrm{Sh}}$ is found to be non linear. The effect of $\mathrm{Ra}$ on $\overline{\mathrm{Nu}}$ is also not significant due to the lower temperature potential between the hot and cold walls $(\Delta \overline{\mathrm{T}}=0.1)$. However Ra has a significant effect on $\overline{\mathrm{Sh}}$ due to the increase in velocity.

Figure 4(f) shows variation of $\overline{\mathrm{Nu}}$ and $\overline{\mathrm{Sh}}$ against $\mathrm{R}$ for various values of $\mathrm{Ra}$ at the cold wall, corresponding to the non uniform temperature cold wall boundary condition with $\lambda=0.25$ (Eq. (17b)). $\overline{\mathrm{Nu}}$ and $\overline{\mathrm{Sh}}$ decrease with $\mathrm{R}$. The variation of $\overline{\mathrm{Nu}}$ and $\overline{\mathrm{Sh}}$ is found to be non linear. However their values in this case lie in between the values corresponding to $\overline{\mathrm{T}}_{\mathrm{o}}=0.9$ and $\overline{\mathrm{T}}_{\mathrm{o}}=0.1$ at the cold wall.

\section{Case 2- Effect of Radiation on $\overline{\mathrm{Nu}}$ and $\overline{\mathrm{Sh}}$}

This case shows variation of $\overline{\mathrm{Nu}}$ and $\overline{\mathrm{Sh}}$ against $\mathrm{R}$ for various values of $\mathrm{Rd}$ at the hot wall and cold wall corresponding to the cold wall temperature boundary conditions. Three different values considered for the radiation parameter in the present case are: 0,5 and 10 .

Figure 5(a) shows variation of $\overline{\mathrm{Nu}}$ and $\overline{\mathrm{Sh}}$ against $\mathrm{R}$ for various values of $\mathrm{Rd}$ at the hot wall, corresponding to the cold wall condition with $\quad \overline{\mathrm{T}}_{\mathrm{o}}=0.1$. It is observed that $\overline{\mathrm{Nu}}$ and $\overline{\mathrm{Sh}}$ increase with $\mathrm{R}$ at the hot surface. The increase in the values of $\overline{\mathrm{Nu}}$ and $\overline{\mathrm{Sh}}$ is due to the effect of reduced thermal and concentration boundary layers, which in turn increase the respective gradients. The increase of $\overline{\mathrm{Nu}}$ and $\overline{\mathrm{Sh}}$ with $\mathrm{R}$ is found to be almost linear. The increase in Rd has a significant effect on $\overline{\mathrm{Nu}}$ as shown in the Figure 5(a). However effect of Rd on $\overline{\mathrm{Sh}}$ is small as expected, as the change in $\overline{\mathrm{Sh}}$ observed is due to the change in velocity only.

Figure 5(b) shows variation of $\overline{\mathrm{Nu}}$ and $\overline{\mathrm{Sh}}$ against $\mathrm{R}$ for various values of $\mathrm{Rd}$ at the hot wall, corresponding to the cold wall condition with $\overline{\mathrm{T}}_{\mathrm{o}}=0.9$. It is observed that $\overline{\mathrm{Nu}}$ and $\overline{\mathrm{Sh}}$ increase with $\mathrm{R}$ at the hot surface. However a reduction is observed in the values of $\overline{\mathrm{Nu}}$ as compared with the case of $\overline{\mathrm{T}}_{\mathrm{o}}=0.1$. The reduction observed is due to the reduced temperature potential between hot wall and the cold wall $(\Delta \overline{\mathrm{T}}=0.1)$. The increase in $\overline{\mathrm{Nu}}$ and $\overline{\mathrm{Sh}}$ with $\mathrm{R}$ is found to be almost 


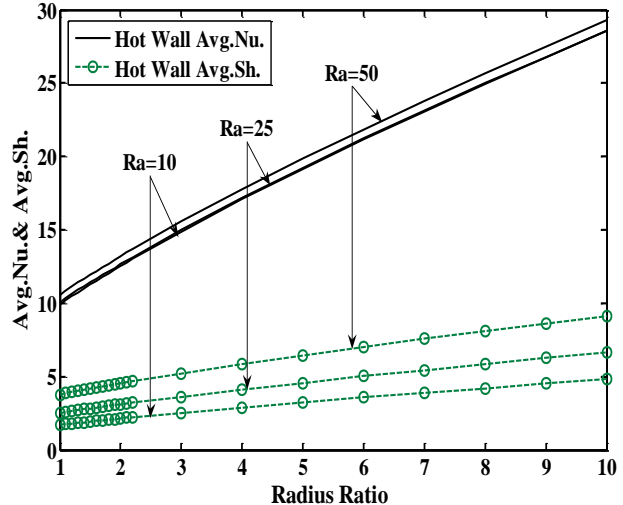

(a)

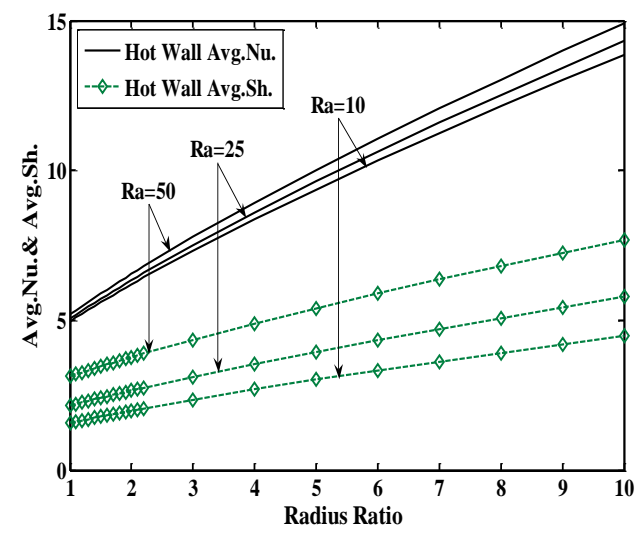

(c)

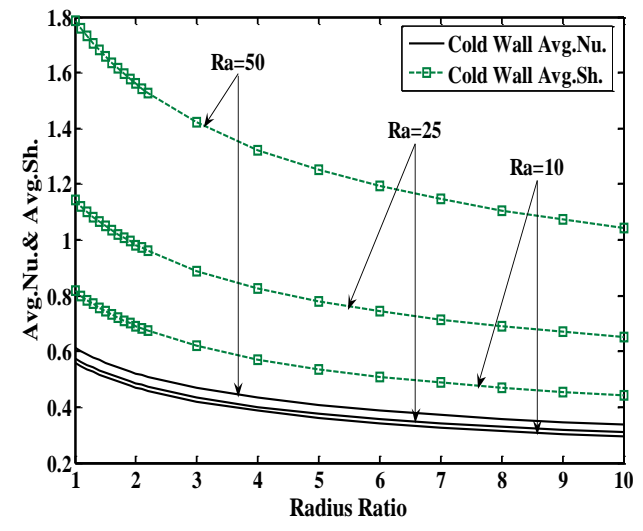

(e)

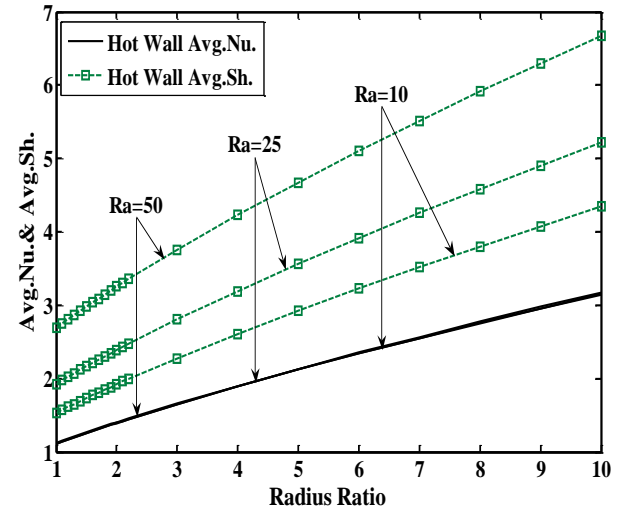

(b)

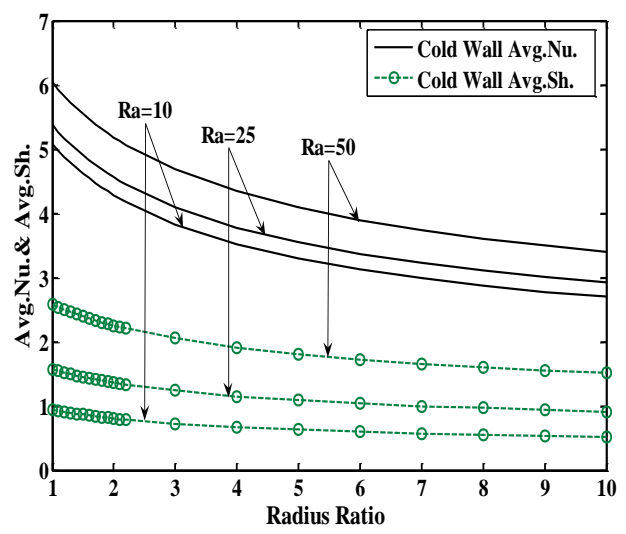

(d)

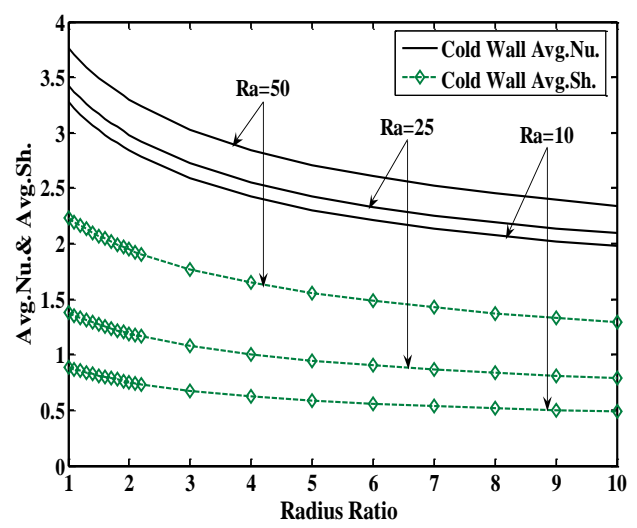

(f)

Fig. 4. Effect of Rayleigh Number on Convection and Mass Transfer (a). (Hot wall) Effect of Ra on $\overline{\mathbf{N u}}$ and $\overline{\mathbf{S h}}$ for $\overline{\mathbf{T}}_{\mathbf{o}}=0.1$.(b). (Hot wall) Effect of Ra on $\overline{\mathbf{N u}}$ and $\overline{\mathbf{S h}}$ for $\overline{\mathbf{T}}_{\mathbf{o}}=0.9$. (c). (Hot wall) Effect of Ra on $\overline{\mathbf{N u}}$ and $\overline{\mathbf{S h}}$ for $\lambda=0.25$. (d). (Cold wall) Effect of Ra on $\overline{\mathbf{N u}}$ and $\overline{\mathbf{S h}}$ for $\overline{\mathbf{T}}_{\mathbf{o}}=0.1$. (e). (Cold wall) Effect of Ra on $\overline{\mathbf{N u}}$ and $\overline{\mathbf{S h}}$ for $\overline{\mathbf{T}}_{\mathbf{o}}=0.9$. (f). (Cold wall) Effect of Ra on $\overline{\mathbf{N u}}$ and $\overline{\mathbf{S h}}$ for $\lambda=0.25$

linear. Increase in Rd has the similar effect on $\overline{\mathrm{Sh}}$ as in in the case of $\overline{\mathrm{T}}_{\mathrm{o}}=0.1$.

Figure 5(c) shows the variation of $\overline{\mathrm{Nu}}$ and $\overline{\mathrm{Sh}}$ against $\mathrm{R}$ for various values of $\mathrm{Rd}$ at the hot wall, corresponding to the non uniform temperature cold wall boundary condition with $\lambda=0.25$ (Eq. (17b)). The variations in
$\overline{\mathrm{Nu}}$ and $\overline{\mathrm{Sh}}$ in the present case are similar to the cases corresponding to $\overline{\mathrm{T}}_{\mathrm{o}}=0.1$ and 0.9 . However their values in this case lie in between the values at hot wall corresponding to $\overline{\mathrm{T}}_{\mathrm{o}}=0.9$ and $\overline{\mathrm{T}}_{\mathrm{o}}=0.1$ at the cold wall.

Figure 5(d) shows the variation of $\overline{\mathrm{Nu}}$ and $\overline{\mathrm{Sh}}$ against $\mathrm{R}$ for various values of $\mathrm{Rd}$ at the cold wall, corresponding 
to the cold wall boundary condition with $\overline{\mathrm{T}}_{\mathrm{O}}=0.1$. It is observed that both $\overline{\mathrm{Nu}}$ and $\overline{\mathrm{Sh}}$ decrease with the increase in R. It is due to the weaker flow region near the cold surface. It is observed that the rate of decrease is more at lower values of $\mathrm{R}$ up to 2 . The variations of $\overline{\mathrm{Nu}}$ and $\overline{\mathrm{Sh}}$ with $\mathrm{R}$ are found to be non linear. The increase in $\mathrm{Rd}$ has a significant effect on $\overline{\mathrm{Nu}}$ and less effect on $\overline{\mathrm{Sh}}$.

Figure 5(e) shows the variation of $\overline{\mathrm{Nu}}$ and $\overline{\mathrm{Sh}}$ against $\mathrm{R}$ for various values of $\mathrm{Rd}$ at the cold wall, corresponding to the cold wall boundary condition with $\overline{\mathrm{T}}_{\mathrm{o}}=0.9$. It is observed that $\overline{\mathrm{Nu}}$ and $\overline{\mathrm{Sh}}$ increase with $\mathrm{R}$ at the cold surface. However a reduction is observed in the values of $\overline{\mathrm{Nu}}$ as compared with the case of $\overline{\mathrm{T}}_{\mathrm{o}}=0.1$. The reduction observed is due to the reduced temperature potential between hot wall and the cold wall The variations of $\overline{\mathrm{Nu}}$ and $\overline{\mathrm{Sh}}$ with $\mathrm{R}$ are found to be non linear. The increase in $\mathrm{Rd}$ has a significant effect on $\overline{\mathrm{Nu}}$ and less effect on $\overline{\mathrm{Sh}}$ as in the case of $\overline{\mathrm{T}}_{\mathrm{o}}=0.1$.

Figure 5(f) shows the variation of $\overline{\mathrm{Nu}}$ and $\overline{\mathrm{Sh}}$ against $\mathrm{R}$ for various values of $\mathrm{Rd}$ at the cold wall, corresponding to the non uniform temperature cold wall boundary condition with $\lambda=0.25$ (Eq. (17b)). The variations in $\overline{\mathrm{Nu}}$ and $\overline{\mathrm{Sh}}$ in the present case are similar to the cases corresponding to $\overline{\mathrm{T}}_{\mathrm{o}}=0.1$ and 0.9 . However their values in this case lie in between the values at cold wall corresponding to $\overline{\mathrm{T}}_{\mathrm{O}}=0.9$ and $\overline{\mathrm{T}}_{\mathrm{o}}=0.1$ at the cold wall.

Figures 6(a) and 6(b) represent the isothermal lines corresponding to $\mathrm{Rd}=0$ and 10 respectively at uniform cold wall temperature boundary condition with $\overline{\mathrm{T}}_{0}=0.1$. As can be seen from these figures, the radiation has a considerable effect on natural convection. At $\mathrm{Rd}=0$, the effect of natural convection is clearly seen as shown in Fig.6(a). Here the convection plays a significant role when the radiation is absent. The thermal gradient is high at the lower corner of the annulus as compared to the other places of the hot surface. The isothermal lines tend to straighten when the radiation increases indicating the lowering effect of convection. The fluid smoothly occupies the entire domain at higher radiation as shown in Fig. 6(b).

Figures 6(c) and 6(d) indicate the cases of isothermal lines corresponding to $\mathrm{Rd}=0$ and 10 respectively at uniform cold wall temperature boundary condition with $\overline{\mathrm{T}}_{\mathrm{o}}=0.9$. The effect of natural convection and radiation is evident in these cases also. The variation in these curves as compared with that of $\bar{T}_{0}=0.1$ is due to the thermal potential between hot and the cold walls.

Figures 6(e) and 6(f) indicate the cases of isothermal lines corresponding to $\mathrm{Rd}=0$ and 10 respectively for the non uniform temperature cold wall boundary condition with $\lambda=0.25$ (Eq. (17b)). The temperature variation in this case is found to be non linear as can be observed from the curves.

Figures 7(a) and 7(b) indicate the cases of stream lines corresponding to $\mathrm{Rd}=0$ and 10 respectively at uniform cold wall temperature boundary condition with $\overline{\mathrm{T}}_{\mathrm{o}}=0.1$. As can be observed from these figures, the increase in radiation has considerable effect on natural convection. The stream lines approach towards circularity as shown in Fig. 7(b).

Figures 7(c) and 7(d) indicate the cases of stream lines corresponding to $\mathrm{Rd}=0$ and 10 respectively at uniform cold wall temperature boundary condition with $\overline{\mathrm{T}}_{\mathrm{o}}=0.9$. The effect of radiation on natural convection is evident in these cases also. The variation in these curves as compared with that of $\overline{\mathrm{T}}_{\mathrm{o}}=0.1$ is due to the thermal potential between hot and the cold walls.

Figures 7 (e) and 7 (f) indicate the cases of stream lines corresponding to $\mathrm{Rd}=0$ and 10 respectively for the non uniform temperature cold wall boundary condition with $\lambda=0.25$ (Eq. (17b)). The effect of natural convection is clearly seen from these figures.

\section{CONCLUSION}

The effect of radiation and the cold wall boundary conditions on the natural convection in a saturated vertical porous medium is considered in the present study.

The cold wall temperature corresponding to nondimensional temperatures of $\overline{\mathrm{T}}_{\mathrm{o}}=0.1,0.4,0.8$ and 0.9 are investigated. However the results for $\overline{\mathrm{T}}_{\mathrm{o}}=0.1$ and 0.9 are only shown in the present work. The governing equations are non-dimensionalised and solved using finite element method.

It is found that the average Nusselt number and average Sherwood number increase with the radius ratio at the hot wall. However they decrease with the increase in radius ratio at the cold wall.

It is also found that the average Nusselt number and average Sherwood number increase with the Rayleigh number at the hot wall and cold wall. The effect of Rayleigh number increases for the case of higher thermal potential between the hot wall and cold wall. Increase in Rayleigh number increases the fluid movement, which is due to the enhanced buoyancy effect. Hence the fluid velocity is increased along with the Nusselt number.

Radiation has a considerable effect on the rise in Nusselt number indicating the increase in natural convection process. 


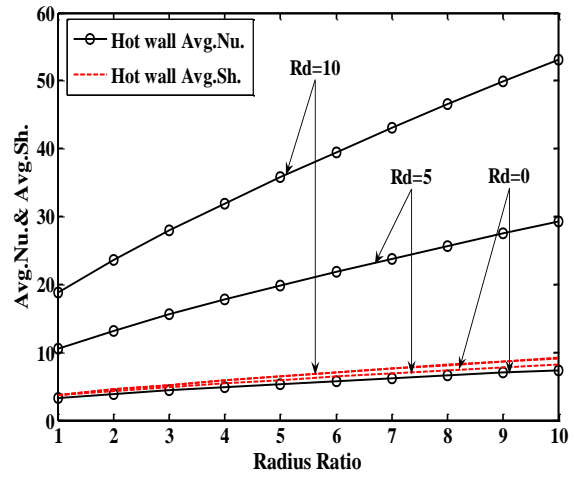

(a)

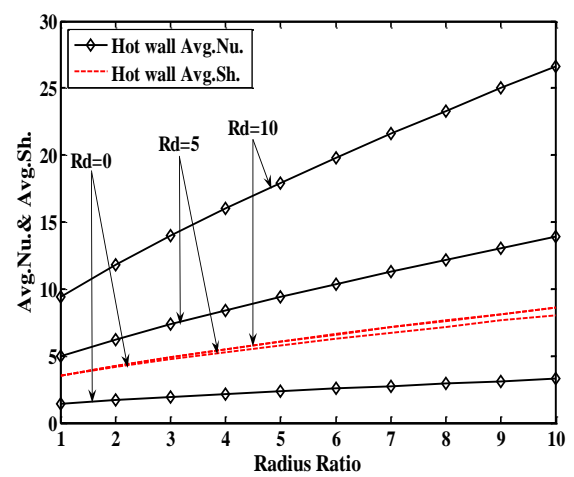

(c)

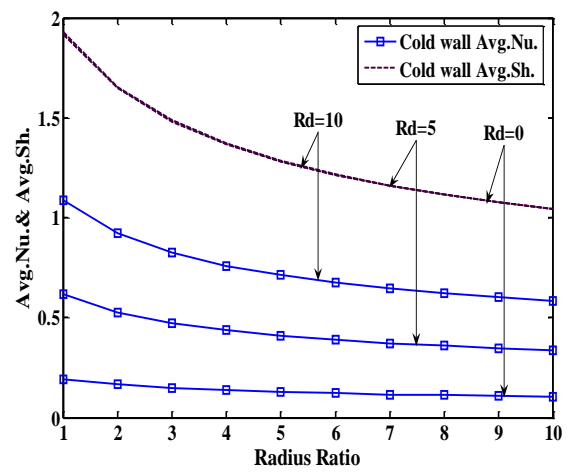

(e)

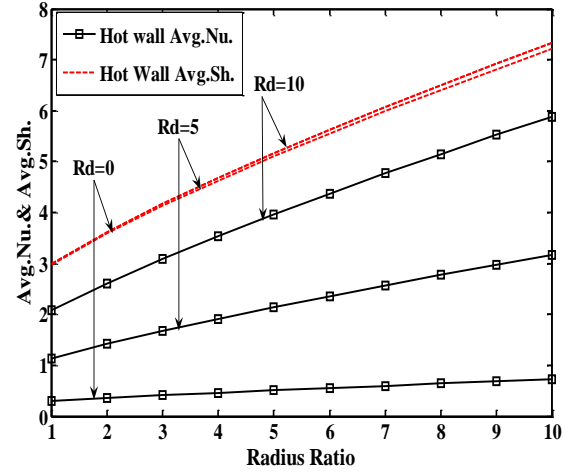

(b)

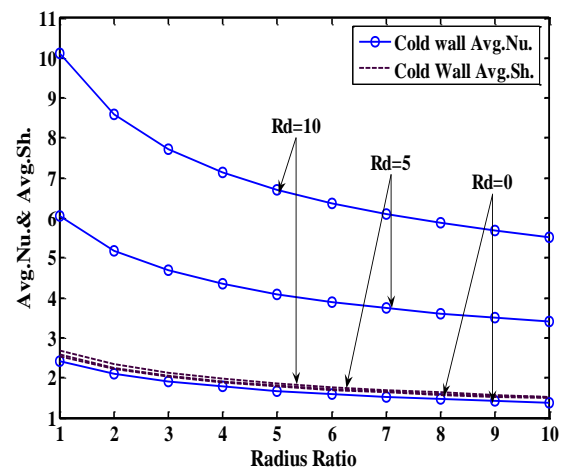

(d)

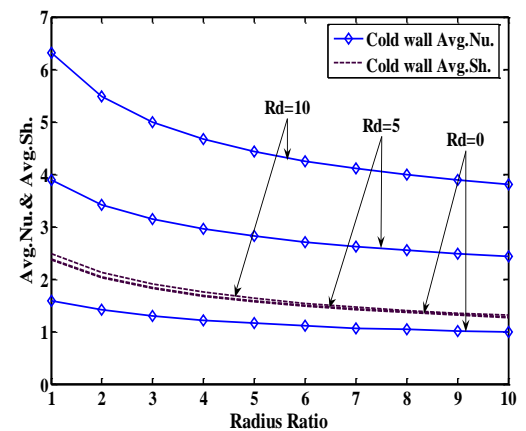

(f)

Fig. 5. Effect of Radiation on Convection and Mass Transfer (a) (Hot wall) Effect of Rd on $\overline{\mathbf{N u}}$ and $\overline{\mathbf{S h}}$ for $\overline{\mathbf{T}}_{\mathbf{0}}=0.1$. (b) (Hot wall) Effect of Rd on $\overline{\mathbf{N u}}$ and $\overline{\mathbf{S h}}$ for $\overline{\mathbf{T}}_{\mathbf{o}}=0.9$.(c) (Hot wall) Effect of Rd on $\overline{\mathbf{N u}}$ and $\overline{\mathbf{S h}}$ for $\lambda=0.25$. (d) Cold wall) Effect of Rd on $\overline{\mathbf{N u}}$ and $\overline{\mathbf{S h}}$ for $\overline{\mathbf{T}}_{\mathbf{o}}=0.1$. (e) (Cold wall) Effect of Rd on $\overline{\mathbf{N u}}$ and $\overline{\mathbf{S h}}$ for $\overline{\mathbf{T}}_{\mathbf{o}}=0.9$. (f) (Cold wall) Effect of Rd on $\overline{\mathbf{N u}}$ and $\overline{\mathbf{S h}}$ for $\lambda=0.25$ Eq. (17b)) 
M. Patil et al. / JAFM, Vol. 6, No. 2, pp. 177-189, 2013.

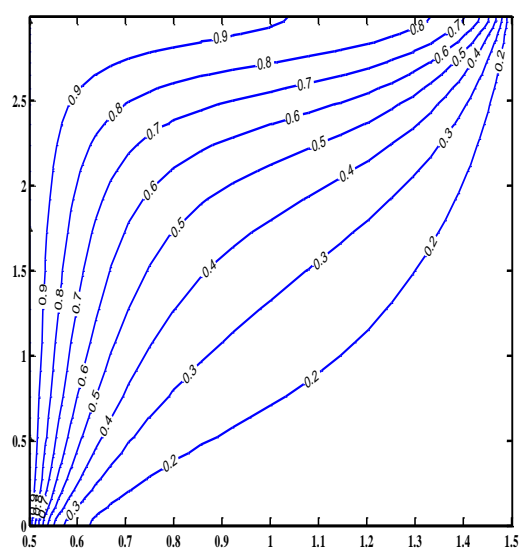

(a)

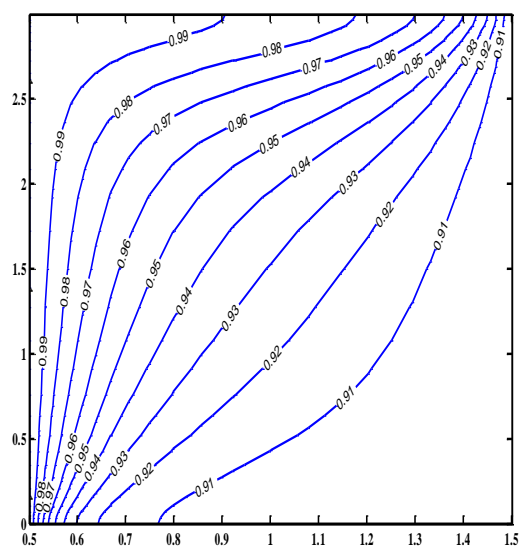

(c)

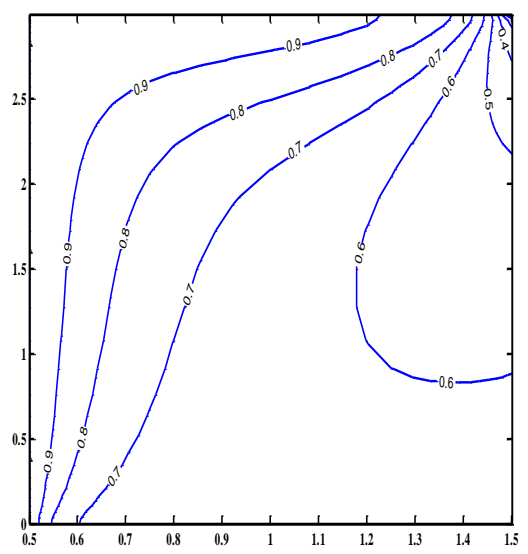

(e)

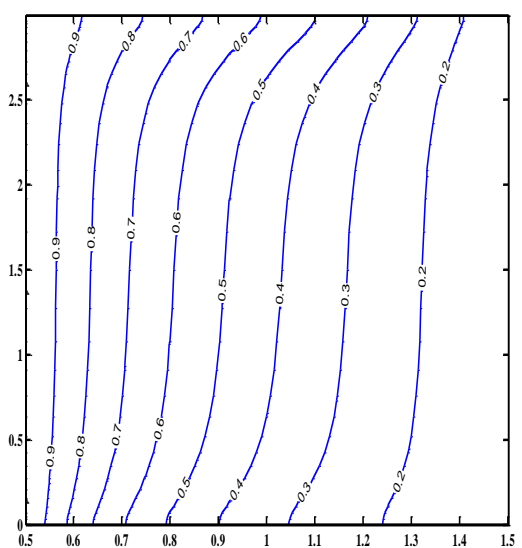

(b)

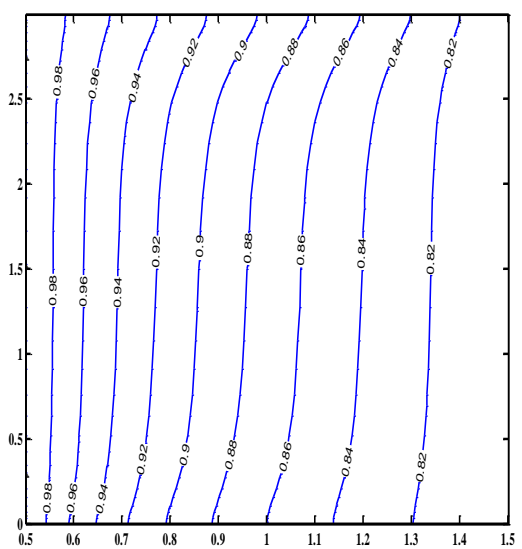

(d)

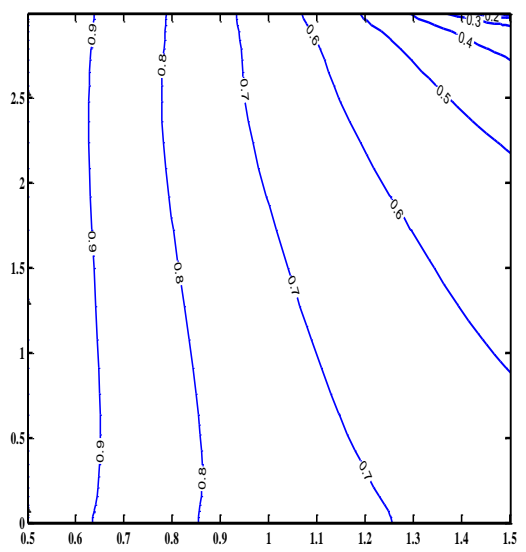

(f)

Fig. 6. Effect of $R d$ on Isothermal lines at $R a=50 ; R=1 ; N=1 ; L e=1$ and $A_{r}=5$ (a) $R d=0$ for $\overline{\mathbf{T}}_{\mathbf{0}}=0.1$. (b). $R d=10$ for $\overline{\mathbf{T}}_{\boldsymbol{o}}=0.1$. (c). $\mathrm{Rd}=0$ for $\overline{\mathbf{T}}_{\mathbf{o}}=0.9$. (d). $\mathrm{Rd}=10$ for $\overline{\mathbf{T}}_{\mathbf{o}}=0.9$. (e). $\mathrm{Rd}=0$ for $\lambda=0.25$. (f). $\mathrm{Rd}=10$ for $\lambda=0.25$ 


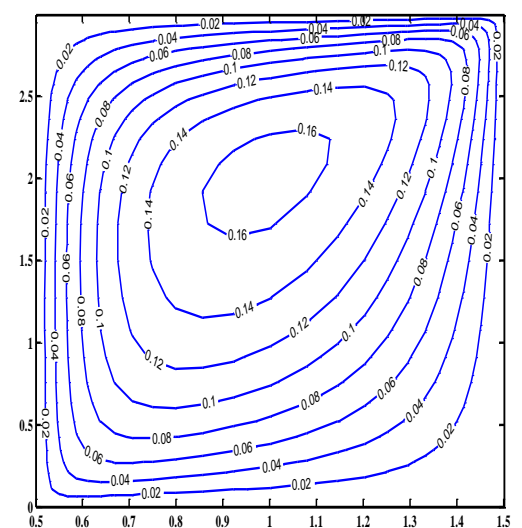

(a)

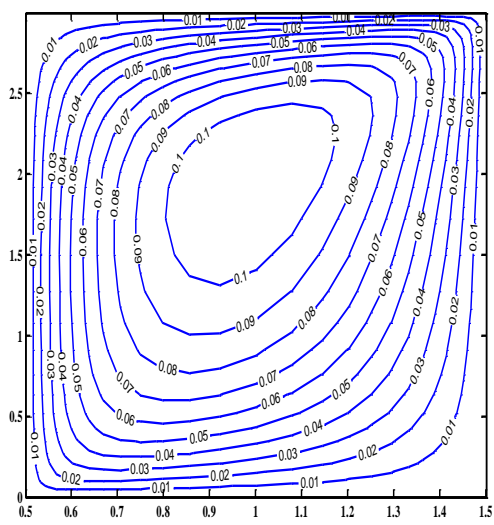

(c)

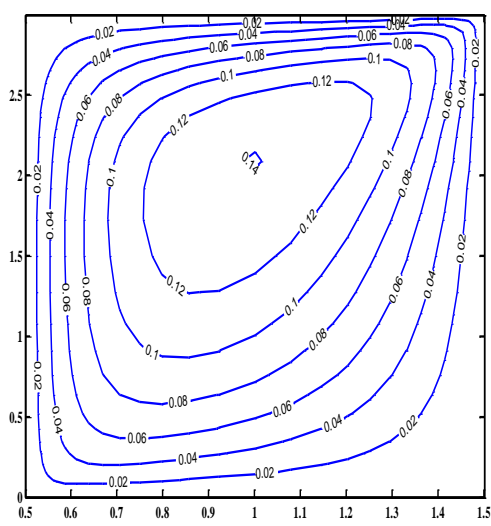

(e)

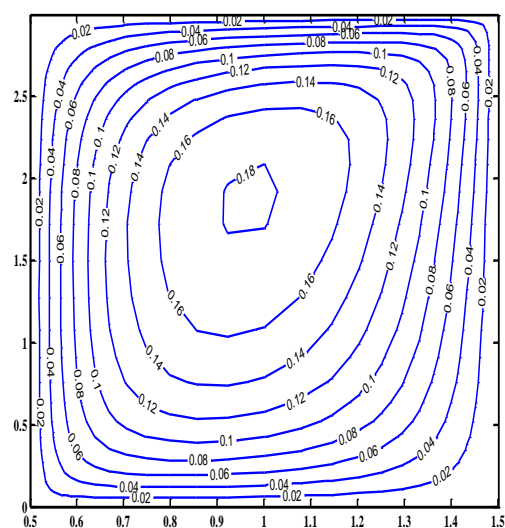

(b)

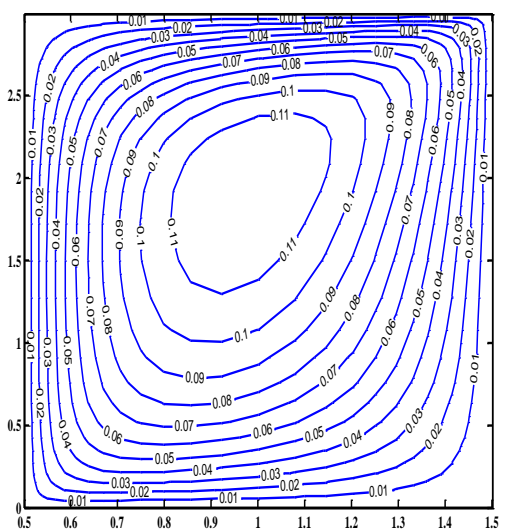

(d)

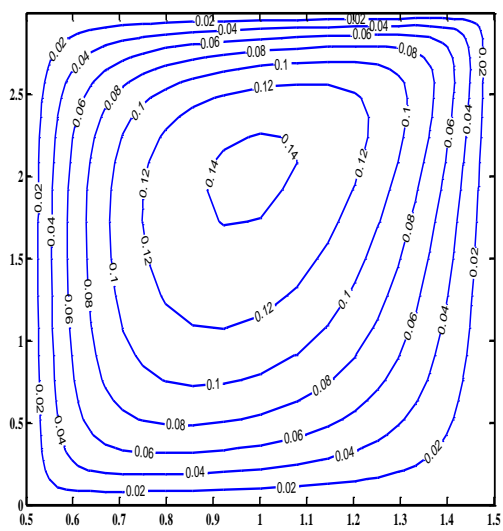

(f)

Fig. 7. Effect of $R d$ on Stream lines at $R a=50 ; R=1 ; N=1 ; L e=1$ and $A_{r}=5$.

(a). $\mathrm{Rd}=0$ for $\overline{\mathbf{T}}_{\mathbf{o}}=0.1$ (b) $\mathrm{Rd}=10$ for $\overline{\mathbf{T}}_{\mathbf{o}}=0.1$. (c). $\mathrm{Rd}=0$ for $\overline{\mathbf{T}}_{\mathbf{o}}=0.9$. (d). $\mathrm{Rd}=10$ for $\overline{\mathbf{T}}_{\mathbf{o}}=0.9$. (e). $\mathrm{Rd}=0$ for $\lambda=0.25$. (f). $\mathrm{Rd}=10$ for $\lambda=0.25$

\section{REFERENCES}

Ahmed, N. J. Salman, Z.A Zainal, Irfan Anjum, Hussain M.T. Khalid (2008). Heat transfer analysis of porous medium in a conical cylinder with variable wall temperature. International Journal of Mechanical and Materials Engineering 3 (2), 145-152.
Cherif, B. and M.S. Sifaoui (2004). Theoretical study of heat transfer by radiation, conduction and convection in a semi transparent porous medium in a cylindrical enclosure. Journal of Quantitative Spectroscopy and Radiative Heat Transfer 83, 519-527. 
Hossain, M.A. and M.A. Alim (1997). Natural convection radiation interaction on boundary layer flow along a thin vertical cylinder. International Journal of Heat Mass Transfer 32, 515-520.

Ingham, D.B. and I. Pop (2005). Transport Phenomena in Porous Media. Oxford, Elsevier.

Irfan, A.B., Z.A. Zainal, P.A. Aswatha Narayana, K.N. Seetharamu (2006). Heat transfer by radiation and natural convection through a vertical cylindrical annular porous medium. International Communications in Heat and Mass Transfer 33, 500-507.

Lewis, R.W., P. Nithiarasu and K.N. Seetharamu, (2004). Fundamentals of the Finite Element Method for Heat and Fluid Flow. John Wiley and Sons.

Muralidhar, K. (1989). Mixed convection flow in a saturated porous annulus. International Journal of Heat and Mass Transfer 32, 881-888.

Nath, S.K. and V.V. Satyamurthy (1985). Effect of aspect ratio and radius ratio on free convection heat transfer in a cylindrical annulus filled with porous media, C16-85, proceedings of 8th National Heat and Mass Transfer Conference, India, pp. 189-193.

Nield, D.A. and A. Bejan (1999). Convection in Porous Media. New York. Springer-Verlag.
Prasad, V. and F.A. Kulacki (1984). Natural convection in a vertical porous annulus. International Journal of Heat and Mass Transfer 27, 207-219.

Raptis (1998). Radiation and free convection flow through porous medium. International journal of Heat and Mass Transfer 25, 289-295.

Rajamani, R.C., C. Srinivas, P. Nithiarasu, K.N. Seetharamu (1995). Convective heat transfer in axisymmetric porous bodies. International Journal of Numerical Methods for Heat and Fluid Flow 5, 829-837.

Reda, D.C. (1986). Natural convection experiments with a finite-length, vertical cylindrical heat source in water saturated porous medium. Nuclear and Chemical Waste Management 6, 3-14.

Segerlind, L.J. (1982). Applied Finite Element Analysis. John Wiley and Sons, New York.

Srivastava, N. and A. K Singh (2010). Mixed convection in a composite system bounded by vertical walls. Journal of Applied Fluid Mechanics $3(2), 65-75$.

Wang, C.Y. (1998). Onset of natural convection in a fluid saturated porous medium inside a cylindrical enclosure bottom heated by constant flux. International Communications in Heat and Mass Transfer 25(4), 593-598.

Yih, K.A. (1999). Radiation effect on natural convection over a vertical cylinder embedded in porous media. International Communications in Heat and Mass Transfer 26(2), 259-267.

\section{APPENDIX}

The matrix form of Eq. (14):

$$
\begin{gathered}
\left.\frac{2 \pi \bar{R}}{4 A}\left[\begin{array}{ccc}
b_{1}^{2} & b_{1} b_{2} & b_{1} b_{3} \\
b_{2} b_{1} & b_{2}^{2} & b_{2} b_{3} \\
b_{3} b_{1} & b_{3} b_{2} & b_{3}^{2}
\end{array}\right]+\left[\begin{array}{ccc}
c_{1}^{2} & c_{1} c_{2} & c_{1} c_{3} \\
c_{2} c_{1} & c_{2}^{2} & c_{2} c_{3} \\
c_{3} c_{1} & c_{3} c_{2} & c_{3}^{2}
\end{array}\right]\right]\left\{\begin{array}{l}
\bar{\Psi}_{1} \\
\bar{\Psi}_{2} \\
\bar{\Psi}_{3}
\end{array}\right\} \\
+2 \pi \bar{R}^{2} R a\left[\begin{array}{l}
b_{1} \bar{T}_{1}+b_{2} \bar{T}_{2}+b_{3} \bar{T}_{3} \\
b_{1} \bar{T}_{1}+b_{2} \bar{T}_{2}+b_{3} \bar{T}_{3} \\
b_{1} \bar{T}_{1}+b_{2} \bar{T}_{2}+b_{3} \bar{T}_{3}
\end{array}\right\}+N\left\{\begin{array}{l}
b_{1} \bar{C}_{1}+b_{2} \bar{C}_{2}+b_{3} \bar{C}_{3} \\
b_{1} \bar{C}_{1}+b_{2} \bar{C}_{2}+b_{3} \bar{C}_{3} \\
b_{1} \bar{C}_{1}+b_{2} \bar{C}_{2}+b_{3} \bar{C}_{3}
\end{array}\right\}=0
\end{gathered}
$$

Where, $\overline{\mathrm{R}}=\frac{\left(\overline{\mathrm{r}}_{1}+\overline{\mathrm{r}}_{2}+\overline{\mathrm{r}}_{3}\right)}{3}$ is the radial distance to the centre of a triangular element.

The matrix form of Eq. (15):

$$
\begin{aligned}
& \frac{2 \pi}{12 \mathrm{~A}}\left\{\begin{array}{l}
c_{1} \bar{\psi}_{1}+c_{2} \bar{\psi}_{2}+c_{3} \bar{\Psi}_{3} \\
c_{1} \bar{\psi}_{1}+c_{2} \bar{\psi}_{2}+c_{3} \bar{\Psi}_{3} \\
c_{1} \bar{\psi}_{1}+c_{2} \bar{\psi}_{2}+c_{3} \bar{\psi}_{3}
\end{array}\right\}\left[\begin{array}{lll}
b_{1} & b_{2} & b_{3}
\end{array}\right]-\frac{2 \pi}{12 \mathrm{~A}}\left\{\begin{array}{c}
b_{1} \bar{\psi}_{1}+b_{2} \bar{\psi}_{2}+b_{3} \bar{\psi}_{3} \\
b_{1} \bar{\psi}_{1}+b_{2} \bar{\psi}_{2}+b_{3} \bar{\psi}_{3} \\
b \bar{\psi}_{1}+b_{2} \bar{\psi}_{2}+b_{3} \bar{\psi}_{3}
\end{array}\right\}\left[\begin{array}{lll}
c_{1} & c_{2} & c_{3}
\end{array}\right]\left\{\begin{array}{l}
\bar{T}_{1} \\
\bar{T}_{2} \\
\bar{T}_{3}
\end{array}\right\} \\
& +\quad \frac{2 \pi \bar{R}}{4 A}\left\{\left(1+\frac{4}{3} R d\right)\left[\begin{array}{ccc}
b_{1}^{2} & b_{1} b_{2} & b_{1} b_{3} \\
b_{2} b_{1} & b_{2}^{2} & b_{2} b_{3} \\
b_{3} b_{1} & b_{3} b_{2} & b_{3}^{2}
\end{array}\right]+\left[\begin{array}{ccc}
c_{1}^{2} & c_{1} c_{2} & c_{1} c_{3} \\
c_{2} c_{1} & c_{2}^{2} & c_{2} c_{3} \\
c_{3} c_{1} & c_{3} c_{2} & c_{3}^{2}
\end{array}\right]\right\}\left\{\begin{array}{l}
\bar{T}_{1} \\
\bar{T}_{2} \\
\bar{T}_{3}
\end{array}\right\}=0
\end{aligned}
$$


M. Patil et al. / JAFM, Vol. 6, No. 2, pp. 177-189, 2013.

The matrix form of Eq. (16):

$$
\begin{gathered}
{\left[\frac{2 \pi}{12 \mathrm{~A}}\left\{\begin{array}{l}
\mathrm{c}_{1} \bar{\psi}_{1}+\mathrm{c}_{2} \bar{\psi}_{2}+\mathrm{c}_{3} \bar{\psi}_{3} \\
\mathrm{c}_{1} \bar{\psi}_{1}+\mathrm{c}_{2} \bar{\psi}_{2}+\mathrm{c}_{3} \bar{\psi}_{3} \\
\mathrm{c}_{1} \bar{\psi}_{1}+\mathrm{c}_{2} \bar{\psi}_{2}+\mathrm{c}_{3} \bar{\psi}_{3}
\end{array}\right\}\left[\begin{array}{lll}
\mathrm{b}_{1} & \mathrm{~b}_{2} & \mathrm{~b}_{3}
\end{array}\right]-\frac{2 \pi}{12 \mathrm{~A}}\left\{\begin{array}{l}
\mathrm{b}_{1} \bar{\psi}_{1}+\mathrm{b}_{2} \bar{\psi}_{2}+\mathrm{b}_{3} \bar{\psi}_{3} \\
\mathrm{~b}_{1} \bar{\psi}_{1}+\mathrm{b}_{2} \bar{\psi}_{2}+\mathrm{b}_{3} \bar{\psi}_{3} \\
\mathrm{~b} \bar{\psi}_{1}+\mathrm{b}_{2} \bar{\psi}_{2}+\mathrm{b}_{3} \bar{\psi}_{3}
\end{array}\right\}\left[\begin{array}{lll}
\mathrm{c}_{1} & \mathrm{c}_{2} & \mathrm{c}_{3}
\end{array}\right]\left\{\begin{array}{l}
\overline{\mathrm{C}}_{1} \\
\overline{\mathrm{C}}_{2} \\
\overline{\mathrm{C}}_{3}
\end{array}\right\}\right.} \\
+\frac{2 \pi \overline{\mathrm{R}}}{4 \mathrm{ALe}}\left\{\left[\begin{array}{ccc}
\mathrm{b}_{1}^{2} & \mathrm{~b}_{1} \mathrm{~b}_{2} & \mathrm{~b}_{1} \mathrm{~b}_{3} \\
\mathrm{~b}_{2} \mathrm{~b}_{1} & \mathrm{~b}_{2}^{2} & \mathrm{~b}_{2} \mathrm{~b}_{3} \\
\mathrm{~b}_{3} \mathrm{~b}_{1} & \mathrm{~b}_{3} \mathrm{~b}_{2} & \mathrm{~b}_{3}^{2}
\end{array}\right]+\left[\begin{array}{ccc}
c_{1}^{2} & c_{1} c_{2} & c_{1} c_{3} \\
c_{2} c_{1} & c_{2}^{2} & c_{2} c_{3} \\
c_{3} c_{1} & c_{3} c_{2} & c_{3}^{2}
\end{array}\right]\right\}\left\{\begin{array}{l}
\bar{C}_{1} \\
\overline{\mathrm{C}}_{2} \\
\overline{\mathrm{C}}_{3}
\end{array}\right\}=0
\end{gathered}
$$

